\title{
Effective Action of QED in Electric Field Backgrounds
}

\author{
Sang Pyo Kim* \\ Department of Physics, Kunsan National University, Kunsan 573-701, Korea and \\ Asia Pacific Center for Theoretical Physics, Pohang 790-784, Korea \\ Hyun Kyu Le屯 $\oplus$ and Yongsung Yoon \\ Department of Physics, Hanyang University, Seoul 133-791, Korea
}

(Dated: October 29, 2018)

\begin{abstract}
We use the evolution operator method to find the one-loop effective action of scalar and spinor QED in electric field backgrounds in terms of the Bogoliubov coefficient between the ingoing and the outgoing vacua. We obtain the exact one-loop effective action for a Sauter-type electric field, $E_{0} \operatorname{sech}^{2}(t / \tau)$, and show that the imaginary part correctly yields the vacuum persistence. The renormalized effective action shows the general relation between the vacuum persistence and the total mean number of created pairs for the constant and the Sauter-type electric field.

PACS numbers: 12.20.-m, 13.40.-f, 12.20.Ds, 11.15.Tk
\end{abstract}

\section{INTRODUCTION}

More than seven decades ago, Sauter, Heisenberg and Euler, and Weisskopf studied the effective action of a charged particle in a constant electromagnetic field [1]. Using the proper time method, Schwinger systematically derived the effective action in a gauge invariant form in a constant electromagnetic field [2]. There have been since then many attempts to find the effective actions in various configurations of electromagnetic fields (for a review, see Ref. [3]). The recent revival of the effective action in strong electromagnetic fields is partly due to a direct test of the strong QED in terrestrial experiments in the near future [4] and partly due to applications to astrophysical objects with strong electromagnetic fields beyond the critical strength.

In contrast to constant electromagnetic fields, finding the effective action in inhomogeneous electromagnetic fields is a non-trivial task. There have been recent attempts to find the pair-production rate in inhomogeneous electric fields, in particular, in localized electric fields either in space or time [5, 6, 7, 8, 9, 10, 11]. A key idea is the electric-magnetic duality of the QED effective action [12], according to which the effective action for a constant magnetic field can be analytically continued to that for a constant electric field. Though a Landau level in a constant magnetic field is dual to the tunneling motion in a constant electric field [13], in the real spacetime a particle accelerates by an electric field, whose unbounded motion makes non-trivial the task of finding the effective action from the time-dependent state. Dunne and Hall used the resolvent technique and directly found the effective action in time-dependent electric fields 14] and Fried and Woodard found the effective action for electric fields that depend on the light-cone time coordinate [15].

Recently, two of us (SKP and HKL) applied the evolution operator method to find the effective action of scalar QED in a constant electric field [16]. In this method the ingoing vacuum evolves to the outgoing vacuum via the evolution operator. The evolution operator is given by a phase part and a squeeze operator, whose parameters are determined by Bogoliubov coefficients. The effective action is then defined through the scattering amplitude between the ingoing and the outgoing vacua [17, 18, 19, 20, 21, 22, 23]. The renormalized effective action for a constant electric field, given by a Bogoliubov coefficient for each momentum, correctly yields not only the vacuum polarization but also the vacuum persistence [16, 18, 20, 21, 22, 23]. However, it is still an open question whether the renormalized effective action for a time-dependent electric field or an inhomogeneous electric field can satisfy the general relation between the vacuum persistence and the total mean number of created pairs.

The main purpose of this paper is to further develop the evolution operator method in scalar QED, first by clarifying the renormalization procedure in a constant electric field and then by applying it to time-dependent electric fields. In particular, we find the exact one-loop effective action for the Sauter-type electric field, $E_{0} \operatorname{sech}^{2}(t / \tau)$, together with or without a constant magnetic field. Finally, we extend the evolution operator method to spinor QED and

\footnotetext{
*Electronic address: sangkim@kunsan.ac.kr

† Electronic address: hyunkyu@hanyang.ac.kr

$\ddagger$ Electronic address: cem@hanyang.ac.kr
} 
find the exact one-loop effective action in the Sauter-type electric field. It is found that our effective action in spinor QED under the Sauter-type electric field shows the correct limiting behaviors, in comparison with the effective action obtained by the resolvent technique [14]. It is further shown that the imaginary parts of the effective action in scalar and spinor QED satisfy the general relations between the vacuum persistence and the total mean number of created scalar particles and fermions.

The organization of this paper is as follows. In Sec. II, we elaborate the evolution operator method for scalar QED by clarifying the renormalization procedure and then extend it to spinor QED. This is done by expressing the Bogoliubov transformation in terms of the evolution operator and then the evolution operator by a two-mode squeeze operator. We find the mean number of created pairs and the vacuum persistence in terms of squeeze parameters, which are, in turn, determined by the Bogoliubov coefficients, and finally obtain the effective action in scalar and spinor QED. In Sec. III, we apply the method to a constant electric field and clarify the renormalization procedure in scalar and spinor QED. In Sec. IV, we find the exact one-loop effective action for a Sauter-type electric field together with or without a constant magnetic field in scalar and spinor QED, which is the main result of this paper.

\section{EFFECTIVE ACTION IN ELECTRIC FIELDS}

The effective action of a charged particle has been studied in various configurations of magnetic fields. The charged particle in a magnetic field has a discrete spectrum from bounded motions, leading to the effective action. However, in a strong electric field, a virtual pair from the Dirac sea gains a sufficient potential energy to separate over the Compton wavelength and to materialize as a real pair. In the time-dependent gauge, the electric field provides a potential barrier, over which the charged particle scatters from an ingoing state to an outgoing one. To find the effective action in electric fields is not straightforward because unbounded motions cause instability of the vacuum. In this section we shall introduce a new method to calculate the effective action at zero temperature using the evolution operator, which can be factored into a phase factor and a squeeze operator.

In the time-dependent gauge, a time-dependent electric field along the $z$-direction is given by $E(t)=-\partial A_{z}(t) / \partial t$. The Fourier component of the Klein-Gordon equation for scalar QED and the spin diagonal component of the Dirac equation for spinor QED satisfy [in units with $\hbar=c=1$ and with metric signature $(+,-,-,-)$ ]

$$
\left[\partial_{t}^{2}+m^{2}+\mathbf{k}_{\perp}^{2}+\left(k_{z}+q A_{z}(t)\right)^{2}+2 i \sigma q E(t)\right] \phi_{\mathbf{k} \sigma}(t)=0
$$

where $\sigma=0$ for scalar particles and $\sigma= \pm 1 / 2$ for spin- $1 / 2$ fermions.

\section{A. Scalar QED}

\section{Evolution Operator in terms of Squeeze Operator}

The ingoing vacuum is annihilated by $a_{\mathbf{k}}\left(t_{\text {in }}=-\infty\right)$ for particle and $b_{\mathbf{k}}\left(t_{\text {in }}=-\infty\right)$ for antiparticle for each momentum, and the outgoing vacuum is similarly defined by $a_{\mathbf{k}}\left(t_{\text {out }}=\infty\right)$ and $b_{\mathbf{k}}\left(t_{\text {out }}=\infty\right)$. These operators are related through the Bogoliubov transformation [24]

$$
\begin{aligned}
& a_{\mathbf{k}, \text { in }}=\mu_{\mathbf{k}}^{*} a_{\mathbf{k}, \text { out }}-\nu_{\mathbf{k}}^{*} b_{\mathbf{k}, \text { out }}^{\dagger}, \\
& b_{\mathbf{k}, \text { in }}=\mu_{\mathbf{k}}^{*} b_{\mathbf{k}, \text { out }}-\nu_{\mathbf{k}}^{*} a_{\mathbf{k}, \text { out }}^{\dagger},
\end{aligned}
$$

where

$$
\begin{aligned}
\mu_{\mathbf{k}} & =i\left(\phi_{\mathbf{k}}^{*}\left(t_{\text {out }}\right) \dot{\phi}_{\mathbf{k}}\left(t_{\text {in }}\right)-\dot{\phi}_{\mathbf{k}}^{*}\left(t_{\text {out }}\right) \phi_{\mathbf{k}}\left(t_{\text {in }}\right)\right) \\
\nu_{\mathbf{k}} & =i\left(\phi_{\mathbf{k}}^{*}\left(t_{\text {out }}\right) \dot{\phi}_{\mathbf{k}}^{*}\left(t_{\text {in }}\right)-\dot{\phi}_{\mathbf{k}}^{*}\left(t_{\text {out }}\right) \phi_{\mathbf{k}}^{*}\left(t_{\text {in }}\right)\right) .
\end{aligned}
$$

with the wronskian condition, $\dot{\phi}_{\mathbf{k}}^{*}(t) \phi_{\mathbf{k}}(t)-\dot{\phi}_{\mathbf{k}}(t) \phi_{\mathbf{k}}^{*}(t)=i$. These coefficients satisfy the relation

$$
\left|\mu_{\mathbf{k}}\right|^{2}-\left|\nu_{\mathbf{k}}\right|^{2}=1 \text {. }
$$

The inverse Bogoliubov transformation is

$$
\begin{aligned}
& a_{\mathbf{k}, \text { out }}=\mu_{\mathbf{k}} a_{\mathbf{k}, \text { in }}+\nu_{\mathbf{k}}^{*} b_{\mathbf{k}, \text { in }}^{\dagger}, \\
& b_{\mathbf{k}, \text { out }}=\mu_{\mathbf{k}} b_{\mathbf{k}, \text { in }}+\nu_{\mathbf{k}}^{*} a_{\mathbf{k}, \text { in }}^{\dagger} .
\end{aligned}
$$


The Bogoliubov coefficients $\mu_{\mathbf{k}}$ and $\nu_{\mathbf{k}}$ implicitly depend on the gauge potential $A_{z}$ through Eq. (1), so they will be denoted by $\mu_{\mathbf{k}}(A)$ and $\nu_{\mathbf{k}}(A)$. For a constant electric field in Sec. III and a Sauter-type electric field in Sec. IV, the coefficients directly found from the exact solution are free of the dynamical phases of evolution. However, a caveat is that the dynamical phase of Eq. (3) should be removed in the effective action to account only interactions with the electric field background. For instance, even a free scalar field for $A_{z}=0$ with the solution $\varphi_{\mathbf{k}}=e^{-i \omega_{\mathbf{k}} t} / \sqrt{2 \omega_{\mathbf{k}}}$, $\left(\omega_{\mathbf{k}}=\sqrt{\mathbf{k}^{2}+m^{2}}\right)$, has a dynamical phase given by

$$
\mu_{\mathbf{k}}(A=0)=e^{i \int_{t_{\text {in }}}^{t_{\text {out }}} \omega_{\mathbf{k}} d t}
$$

and $\nu_{\mathbf{k}}=0$. Thus, the Bogoliubov coefficients in Eq. (3) carry the information of both the dynamical phase and the gauge potential.

To express the outgoing vacuum as multi-particle states of the ingoing vacuum, we write the Bogoliubov transformation (5) as a unitary transformation

$$
\begin{aligned}
a_{\mathbf{k}, \text { out }} & =U_{\mathbf{k}} a_{\mathbf{k}, \text { in }} U_{\mathbf{k}}^{\dagger}, \\
b_{\mathbf{k}, \text { out }} & =U_{\mathbf{k}} b_{\mathbf{k}, \text { in }} U_{\mathbf{k}}^{\dagger} .
\end{aligned}
$$

Here, the evolution operator,

$$
U_{\mathbf{k}}(A)=S_{\mathbf{k}}(A) P_{\mathbf{k}}(A)
$$

is factored by the overall phase factor and the two-mode squeeze operator [25, 26]

$$
\begin{aligned}
& P_{\mathbf{k}}=\exp \left[i \theta_{\mathbf{k}}\left(a_{\mathbf{k}, \text { in }}^{\dagger} a_{\mathbf{k}, \text { in }}+b_{\mathbf{k}, \text { in }}^{\dagger} b_{\mathbf{k}, \text { in }}+1\right)\right] \\
& S_{\mathbf{k}}=\exp \left[r_{\mathbf{k}}\left(a_{\mathbf{k}, \text { in }} b_{\mathbf{k}, \text { in }} e^{-2 i \vartheta_{\mathbf{k}}}-a_{\mathbf{k}, \text { in }}^{\dagger} b_{\mathbf{k}, \text { in }}^{\dagger} e^{2 i \vartheta_{\mathbf{k}}}\right)\right],
\end{aligned}
$$

where the squeeze parameter $r_{\mathbf{k}}$, the squeeze angle $\vartheta_{\mathbf{k}}$, and the overall phase angle $\theta_{\mathbf{k}}$ are determined by

$$
\begin{aligned}
\mu_{\mathbf{k}} & =e^{-i \theta_{\mathbf{k}}} \cosh r_{\mathbf{k}}, \\
\nu_{\mathbf{k}}^{*} & =-e^{-i \theta_{\mathbf{k}}}\left(e^{2 i \vartheta_{\mathbf{k}}} \sinh r_{\mathbf{k}}\right) .
\end{aligned}
$$

As the outgoing vacuum is the two-mode squeezed vacuum

$$
\left.\left.\mid 0, \text { out }\rangle=\prod_{\mathbf{k}} U_{\mathbf{k}}(A) \mid 0, \text { in }\right\rangle=U(A) \mid 0, \text { in }\right\rangle,
$$

the scattering amplitude is given by

$$
\langle 0, \text { out }| 0, \text { in }\rangle=\left\langle 0, \text { in }\left|U^{\dagger}\right| 0, \text { in }\right\rangle=\prod_{\mathbf{k}} e^{i \theta_{\mathbf{k}}}\left\langle 0, \text { in }\left|S_{\mathbf{k}}^{\dagger}\right| 0, \text { in }\right\rangle .
$$

The squeeze operator can further be factored as [26]

$$
S_{\mathbf{k}}=\exp \left[\xi_{\mathbf{k}} a_{\mathbf{k}, \text { in }}^{\dagger} b_{\mathbf{k}, \text { in }}^{\dagger}\right] \exp \left[\frac{\gamma_{\mathbf{k}}}{2}\left(a_{\mathbf{k}, \text { in }}^{\dagger} a_{\mathbf{k}, \text { in }}+b_{\mathbf{k}, \text { in }}^{\dagger} b_{\mathbf{k}, \text { in }}+1\right)\right] \exp \left[-\xi_{\mathbf{k}}^{*} a_{\mathbf{k}, \text { in }} b_{\mathbf{k}, \text { in }}\right]
$$

where

$$
\begin{aligned}
\xi_{\mathbf{k}} & =-e^{2 i \vartheta_{\mathbf{k}}} \tanh r_{\mathbf{k}} \\
\gamma_{\mathbf{k}} & =\ln \left(1-\left|\xi_{\mathbf{k}}\right|^{2}\right)=-2 \ln \left(\cosh r_{\mathbf{k}}\right)
\end{aligned}
$$

\section{Mean Number of Pairs and Vacuum Persistence}

Using the squeeze operator, we now find the vacuum persistence and the mean number of created pairs. Due to charge neutrality, the multi-particle state for $n$-pairs consists of equal number of particles and antiparticles, which is compactly denoted as $\left|n_{\mathbf{k}}, t\right\rangle=\left(a_{\mathbf{k}}^{\dagger n}(t) / \sqrt{n !}\right)\left(b_{\mathbf{k}}^{\dagger n}(t) / \sqrt{n !}\right)|0 ; t\rangle$. The probability for $n$-pairs with momentum $\mathbf{k}$ to be created from the vacuum is

$$
\left.P_{n}(\mathbf{k})=\mid\left\langle n_{\mathbf{k}}, \text { out }\right| 0, \text { in }\right\rangle\left.\right|^{2}=\mid\left.\left\langle n_{\mathbf{k}}, \text { in }\left|S_{\mathbf{k}}^{\dagger}\right| 0, \text { in }\right\rangle\right|^{2}=e^{\gamma_{\mathbf{k}}}\left|\xi_{\mathbf{k}}\right|^{2 n} .
$$


Note that $P_{0}=e^{\gamma_{\mathbf{k}}}$ and $P_{1}=e^{\gamma_{\mathbf{k}}}\left|\xi_{\mathbf{k}}\right|^{2}$ so that $P_{n}=P_{0}\left(P_{1} / P_{0}\right)^{n}$ and $\sum_{n=0}^{\infty} P_{n}=1$ for each $\mathbf{k}$. Thus, the mean number of pairs created from the vacuum for each momentum per unit volume is

$$
\mathcal{N}_{\mathbf{k}}=\sum_{n=0}^{\infty} n P_{n}(\mathbf{k})=\sinh ^{2} r_{\mathbf{k}}=\left|\nu_{\mathbf{k}}\right|^{2} .
$$

The vacuum persistence is

$$
\mid\langle 0, \text { out }| 0, \text { in }\rangle\left.\right|^{2}=\prod_{\mathbf{k}} P_{0}(\mathbf{k})=\prod_{\mathbf{k}} \frac{1}{\cosh ^{2} r_{\mathbf{k}}}=\prod_{\mathbf{k}} \frac{1}{\left|\mu_{\mathbf{k}}\right|^{2}} .
$$

Alternatively, the vacuum persistence is the probability for the ingoing vacuum to remain the outgoing vacuum

$$
\mid\langle 0, \text { out }| 0, \text { in }\rangle\left.\right|^{2}=\prod_{\mathbf{k}}\left[1-\sum_{n=1}^{\infty} P_{n}(\mathbf{k})\right]=\prod_{\mathbf{k}} \frac{1}{\cosh ^{2} r_{\mathbf{k}}} .
$$

\section{Effective Action}

Following Refs. 16, 17, 18, 19, 20, 21, 22, 23], the effective action at zero temperature is defined by the scattering amplitude as

$$
\left.e^{i S_{\text {eff }}^{\mathrm{sc}}}=e^{i \int d t d^{3} \mathbf{x} \mathcal{L}_{\text {eff }}^{\mathrm{sc}}}=\langle 0, \text { out }| 0, \text { in }\right\rangle .
$$

In fact, the effective action is equivalent to the usual form

$$
e^{i S_{\mathrm{eff}}^{\mathrm{sc}}}=\int \mathcal{D} \phi^{*} \mathcal{D} \phi e^{i \int \mathcal{L}^{\mathrm{sc}}(A)} .
$$

After a gymnastic of algebra, the effective action is given by

$$
\langle 0, \text { out }| 0, \text { in }\rangle=\prod_{\mathbf{k}} \frac{1}{\mu_{\mathbf{k}}^{*}} .
$$

The effective action may be found directly from the ingoing and the outgoing vacuum wave functional, for instance, from the ground state for $a_{\mathbf{k}}$ and $b_{\mathbf{k}}$,

$$
\left.\left\langle 0_{\mathbf{k}}, \text { out }\right| 0_{\mathbf{k}}, \text { in }\right\rangle=\left[\frac{\varphi_{\mathbf{k}}^{*}\left(t_{\text {in }}\right) \varphi_{\mathbf{k}}\left(t_{\text {out }}\right)}{\left|\varphi_{\mathbf{k}}^{*}\left(t_{\text {in }}\right) \varphi_{\mathbf{k}}\left(t_{\text {out }}\right)\right|}\right] \frac{1}{\mu_{\mathbf{k}}^{*}} .
$$

Here, the dynamical phase in the square bracket, which is, for instance, $e^{-i \int_{t_{\mathrm{in}}}^{t_{\text {out }}} \omega_{\mathbf{k}} d t}$ for $A=0$, is canceled by that of $\mu_{\mathbf{k}}^{*}$, so the effective action (22) is independent of the dynamical phase, as in Eq. (21).

Finally, we find the effective action per unit volume

$$
\mathcal{L}_{\mathrm{eff}}^{\mathrm{sc}}=i \sum_{\mathbf{k}} \ln \left(\mu_{\mathbf{k}}^{*}\right)
$$

where the summation is over all possible states in the momentum space. It can be shown that the vacuum persistence

$$
\mid\langle 0, \text { out }| 0, \text { in }\rangle\left.\right|^{2}=e^{-2\left(\operatorname{Im} \mathcal{S}_{\text {eff }}^{\mathrm{sc}}\right)}=e^{-V \sum_{\mathbf{k}} \ln \left(1+\mathcal{N}_{\mathbf{k}}^{\mathrm{sc}}\right)},
$$

where $V$ is the volume, leads to the exact relation between the imaginary part and the total mean number of created scalar pairs

$$
2\left(\operatorname{Im} \mathcal{L}_{\text {eff }}^{\mathrm{sc}}\right)=\sum_{\mathbf{k}} \ln \left(1+\mathcal{N}_{\mathbf{k}}^{\mathrm{sc}}\right) .
$$

The relation (25) is generally true for any electric field background. In the weak-field limit, $\left(\left|\nu_{\mathbf{k}}\right|^{2} \ll 1\right)$, twice of the imaginary part of the effective action per unit volume is the total mean number of created pairs, $2\left(\operatorname{Im} \mathcal{L}_{\text {eff }}^{\text {sc }}\right) \approx$ $\sum_{\mathbf{k}} \mathcal{N}_{\mathbf{k}}^{\mathrm{sc}}=\mathcal{N}^{\mathrm{sc}}$.

However, it should be pointed out that the effective action (23) and thereby the general relation (25) involve diverging terms, which require some proper regularization scheme to yield renormalized ones. In this paper we shall show that the renormalized effective action in scalar QED indeed satisfies the general relation (25) for a constant and a Sauter-type time-dependent electric field. 


\section{B. Spinor QED}

In spinor QED, the Bogoliubov transformation between the ingoing and the outgoing particle and antiparticle operators, $b_{n}, d_{n}$, is given by

$$
\begin{aligned}
& b_{n, \text { out }}=\mu_{n} b_{n, \text { in }}+i \nu_{n}^{*} d_{n, \text { in }}^{\dagger}, \\
& d_{n, \text { out }}=\mu_{n} d_{n, \text { in }}-i \nu_{n}^{*} b_{n, \text { in }}^{\dagger}
\end{aligned}
$$

where $n=(\mathbf{k}, \sigma)$ denotes the momentum and spin states, $\sigma= \pm 1 / 2$. The Bogoliubov coefficients satisfy the relation

$$
\left|\mu_{n}\right|^{2}+\left|\nu_{n}\right|^{2}=1
$$

The Bogoliubov transformation can be written as a unitary transformation [27]

$$
\begin{aligned}
& b_{n, \text { out }}=U_{n} b_{n, \text { in }} U_{n}^{\dagger}, \\
& d_{n, \text { out }}=U_{n} d_{n, \text { in }} U_{n}^{\dagger},
\end{aligned}
$$

where the evolution operator is factored into the overall phase factor and the two-mode squeeze operator for fermions as

$$
U_{n}=e^{\xi_{n} b_{n, \text { in }}^{\dagger} d_{n, \text { in }}^{\dagger}} e^{\left(\frac{\gamma_{n}}{2}+i \theta_{n}\right)\left(b_{n, \text { in }}^{\dagger} b_{n, \text { in }}+d_{n, \text { in }}^{\dagger} d_{n, \text { in }}-1\right)} e^{e^{2 i \theta_{n}} \xi_{n}^{*} b_{n, \text { in }} d_{n, \text { in }}}
$$

The Bogoliubov coefficients are determined by three real parameters $\theta_{n}, \vartheta_{n}, r_{n}$ for the evolution operator as follows

$$
\begin{aligned}
\mu_{n} & =e^{-i \theta_{n}} \cos r_{n}, \\
\nu_{n}^{*} & =-e^{-i \theta_{n}}\left(e^{2 i \vartheta_{n}} \sin r_{n}\right) \\
\gamma_{n} & =-2 \ln \left(\cos r_{n}\right), \\
\xi_{n} & =i e^{2 i \vartheta_{n}} \tan r_{n} .
\end{aligned}
$$

The effective action defined as the scattering amplitude,

$$
e^{i S_{\mathrm{eff}}^{\mathrm{sp}}}=\left\langle 0, \text { in }\left|\prod_{n} U_{n}^{\dagger}\right| 0, \text { in }\right\rangle,
$$

leads, with the aid of Eq. (29), to the effective action per unit volume for spinor QED in the form

$$
\mathcal{L}_{\mathrm{eff}}^{\mathrm{sp}}=-i \sum_{n} \ln \left(\mu_{n}^{*}\right)
$$

where the summation $n$ is over all possible momentum $\mathbf{k}$ and spin $\sigma$. The mean number of pairs created from the vacuum for each state $n$ is calculated as

$$
\left.\mathcal{N}_{n}^{\text {sp }}=\mid\left\langle 1_{n}, \text { out }\right| 0, \text { in }\right\rangle\left.\right|^{2}=\sin ^{2} r_{n}=1-\left|\mu_{n}\right|^{2} .
$$

Therefore, the vacuum persistence

$$
\mid\langle 0, \text { out }| 0, \text { in }\rangle\left.\right|^{2}=e^{-2\left(\operatorname{Im} \mathcal{S}_{\text {eff }}^{\mathrm{sp}}\right)}=e^{V \sum_{n} \ln \left(1-\mathcal{N}_{n}^{\mathrm{sp}}\right)},
$$

where $V$ is the volume, leads to the exact relation between the imaginary part and the total mean number of created pairs

$$
2\left(\operatorname{Im} \mathcal{L}_{\text {eff }}^{\mathrm{sp}}\right)=-\sum_{n} \ln \left(1-\mathcal{N}_{n}^{\mathrm{sp}}\right) .
$$

The relation (35) is generally true for any electric field background in spinor QED.

As in the case of scalar QED, the spinor effective action (32) and the general relation (35) are not renormalized yet. In the next sections we shall put forth a regularization scheme, obtain the renormalized effective action and show the general relation (35). 


\section{EFFECTIVE ACTION IN A CONSTANT ELECTRIC FIELD}

As the first application of the method in Sec. II, we find the effective action for a constant electric field. In the time-dependent gauge $A_{z}=-E t$, the Fourier-component of the Klein-Gordon or the spin diagonal Dirac equation takes the form

$$
\left[\partial_{t}^{2}+m^{2}+\mathbf{k}_{\perp}^{2}+\left(k_{z}-q E t\right)^{2}+2 i \sigma q E\right] \phi_{\omega, \mathbf{k}}(t)=0,
$$

where $\sigma=0$ for scalar QED and $\sigma= \pm 1 / 2$ for spinor QED. The equation describes the scattering problem over an inverted harmonic potential. The asymptotic ingoing and the outgoing vacuum may be defined with respect to the asymptotically positive frequency at $t=-\infty$ and $+\infty$, respectively. A positive frequency solution at $t=-\infty$ is, in general, scattered into a branch of positive frequency and another branch of negative frequency at $t=\infty$. The solution of the parabolic cylinder function

$$
\phi_{\omega, \mathbf{k}}(t)=D_{p}(z)
$$

with

$$
z=\sqrt{\frac{2}{q E}} e^{i \pi / 4}\left(k_{z}-q E t\right), \quad p=-\frac{1}{2}-i \frac{m^{2}+\mathbf{k}_{\perp}^{2}+2 i \sigma q E}{2(q E)},
$$

has an appropriate ingoing flux at $t=-\infty$ with respect to the asymptotic form $D_{p}(z) \approx e^{-z^{2} / 4} z^{p}$ for $|z| \gg 1$. In the other asymptotic region at $t=\infty$, the solution is analytically continued to the form [28]

$$
D_{p}(z)=e^{-i p \pi} D_{p}(-z)+\frac{\sqrt{2 \pi}}{\Gamma(-p)} e^{-i(p+1) \pi / 2} D_{-p-1}(i z) .
$$

Thus, we find the Bogoliubov coefficients

$$
\mu_{\mathbf{k}}=\frac{\sqrt{2 \pi}}{\Gamma(-p)} e^{-i(p+1) \pi / 2}, \quad \nu_{\mathbf{k}}=e^{-i p \pi}
$$

\section{A. Scalar QED}

From Eq. (23) for scalar QED, the effective action per unit volume is given by

$$
\mathcal{L}_{\mathrm{eff}}^{\mathrm{sc}}=i \frac{q E}{2 \pi} \int \frac{d^{2} \mathbf{k}_{\perp}}{(2 \pi)^{2}}\left[\ln \sqrt{2 \pi}-\ln \Gamma\left(-p^{*}\right)-i \frac{\left(p^{*}+1\right) \pi}{2}\right] .
$$

Here, $q E /(2 \pi)$ is the number of states along the $z$-direction and $d^{2} \mathbf{k}_{\perp} /(2 \pi)^{2}$ is the number of states for each $\mathbf{k}_{\perp}$. Using the gamma function [29]

$$
\ln \Gamma(z)=\int_{0}^{\infty}\left[\frac{e^{-z s}}{1-e^{-s}}-\frac{e^{-s}}{1-e^{-s}}+(z-1) e^{-s}\right] \frac{d s}{s},
$$

and subtracting all divergent terms which are independent of $q E$ and linear in $q E$, the effective action per unit volume takes the form

$$
\mathcal{L}_{\text {eff }}^{\mathrm{sc}}=-i \frac{q E}{4 \pi} \int \frac{d^{2} \mathbf{k}_{\perp}}{(2 \pi)^{2}} \int_{0}^{\infty} \frac{d s}{s} e^{\left(p^{*}+1 / 2\right) s}\left[\frac{1}{\sinh (s / 2)}-\frac{2}{s}+\frac{s}{12}\right] .
$$

Note that the effective action (43) is now finite after a renormalization prescription, where the (2/s)-subtraction, independent of $q E$, corresponds to the vacuum-energy renormalization, and the $(-s / 12)$-subtraction, quadratic in $q E$, corresponds to the charge renormalization. Also, note that the resonances at $p^{*}=n$ of scattering amplitude, $1 / \mu_{\mathbf{k}}^{*}$, which correspond to the complex energy eigenvalues, $m^{2}+\mathbf{k}_{\perp}^{2}+i q E(2 n+1)$, from the electric-magnetic duality in Ref. [14], are regular points of the integral in Eq. (43).

Further, by integrating over the momentum $\mathbf{k}_{\perp}$, the effective action is given by a finite integral

$$
\mathcal{L}_{\mathrm{eff}}^{\mathrm{sc}}=\frac{1}{16 \pi^{2}} \int_{0}^{\infty} \frac{d s}{s^{3}} e^{i m^{2} s}\left[\frac{q E s}{\sinh (q E s)}-1+\frac{(e E s)^{2}}{6}\right] .
$$




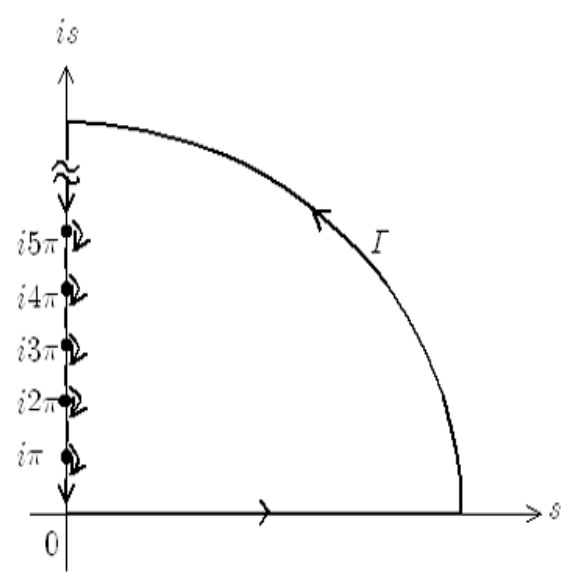

FIG. 1: The contour of $s$ integration for Eq. (50)

Finally, by doing the contour integral over a quarter circle in the first quadrant in Fig. 1, we obtain the exact one-loop effective action per unit volume

$$
\mathcal{L}_{\mathrm{eff}}^{\mathrm{sc}}=-\frac{1}{16 \pi^{2}} \mathcal{P} \int_{0}^{\infty} \frac{d s}{s^{3}} e^{-m^{2} s}\left[\frac{q E s}{\sin (q E s)}-1-\frac{(e E s)^{2}}{6}\right]+i \frac{(q E)^{2}}{16 \pi^{3}} \sum_{n=1}^{\infty} \frac{(-1)^{n+1}}{n^{2}} e^{-\frac{\pi m^{2} n}{q E}},
$$

where $\mathcal{P}$ denotes the principal value. The effective action (45) agrees with the exact result by Schwinger [2]. The imaginary part may be written in the form

$$
\operatorname{Im}\left(\mathcal{L}_{\text {eff }}^{\mathrm{sc}}\right)=\frac{q E}{2(2 \pi)} \int \frac{d^{2} \mathbf{k}_{\perp}}{(2 \pi)^{2}} \ln \left(1+\mathcal{N}_{\mathbf{k}}\right)
$$

where $\mathcal{N}_{\mathbf{k}}=e^{-\pi\left(m^{2}+\mathbf{k}_{\perp}^{2}\right) /(q E)}$, and confirms the relation (25), now renormalized one.

A passing remark is that the Bogoliubov coefficients (40) can also be obtained from Eq. (3) by appropriately normalizing the ingoing wave function as

$$
\varphi_{\mathbf{k}, \text { in }}(t)=\frac{e^{-\pi \frac{m^{2}+\mathbf{k}_{\perp}^{2}}{4 q E}}}{\sqrt{2 q E}} D_{p}(z),
$$

and by choosing $t_{\text {in }}+t_{\text {out }}=2 k_{z} / q E$, which amounts to removing the dynamical phase.

\section{B. Spinor QED}

In spinor QED, the effective action per unit volume can be obtained from Eq. (32) as

$$
\mathcal{L}_{\mathrm{eff}}^{\mathrm{sp}}=-i \frac{q E}{2 \pi} \int \frac{d^{2} \mathbf{k}_{\perp}}{(2 \pi)^{2}}\left[\ln \sqrt{2 \pi}-\ln \Gamma\left(-p^{*}\right)-i \frac{\left(p^{*}+1\right) \pi}{2}\right] .
$$

Using the gamma function (42) and summing over the spin states $(\sigma= \pm 1 / 2)$, we find the effective action per unit volume in the form

$$
\mathcal{L}_{\mathrm{eff}}^{\mathrm{sp}}=-i \frac{q E}{4 \pi} \int \frac{d^{2} \mathbf{k}_{\perp}}{(2 \pi)^{2}} \int_{0}^{\infty} \frac{d s}{s} e^{i \frac{m^{2}+\mathbf{k}_{\perp}^{2}}{2 q E} s}\left[\operatorname{coth}(s / 2)-\frac{2}{s}+\frac{s}{12}\right]
$$

Finally, integrating over the momentum $\mathbf{k}_{\perp}$ and doing the contour integral as in the case of scalar QED, we obtain the renormalized effective action

$$
\mathcal{L}_{\text {eff }}^{\mathrm{sp}}=\frac{1}{8 \pi^{2}} \mathcal{P} \int_{0}^{\infty} \frac{d s}{s^{3}} e^{-m^{2} s}\left[(q E s) \cot (q E s)-\frac{1}{s}+\frac{(e E s)^{3}}{3}\right]+i \frac{(q E)^{2}}{8 \pi^{3}} \sum_{n=1}^{\infty} \frac{1}{n^{2}} e^{-\frac{\pi m^{2} n}{q E}}, .
$$


The effective action (50) agrees with the exact result by Schwinger [2]. The imaginary part may be written in the form

$$
\operatorname{Im}\left(\mathcal{L}_{\mathrm{eff}}^{\mathrm{sp}}\right)=-\frac{q E}{(2 \pi)} \int \frac{d^{2} \mathbf{k}_{\perp}}{(2 \pi)^{2}} \ln \left(1-\mathcal{N}_{\mathbf{k}}\right)
$$

where $\mathcal{N}_{\mathbf{k}}=e^{-\pi\left(m^{2}+\mathbf{k}_{\perp}^{2}\right) /(q E)}$, and confirms the relation (35), which is now renormalized.

\section{EFFECTIVE ACTION IN A SAUTER-TYPE ELECTRIC FIELD}

In this section we turn to the main result of this paper, the exact one-loop effective action for a Sauter-type electric field $E(t)=E_{0} \operatorname{sech}^{2}(t / \tau)[30]$ together with or without a parallel constant magnetic field. In a Lorentz frame where the electric field is parallel to the magnetic field, we may choose as

$$
A_{\mu}=\left(0,-\frac{B y}{2}, \frac{B x}{2},-E_{0} \tau\left(1+\tanh \left(\frac{t}{\tau}\right)\right)\right) .
$$

Here, we have chosen the gauge such that the Klein-Gordon or Dirac equation reduces to a free scalar theory in the past infinity $(t=-\infty)$ when $B=0$. In the first case of a pure electric field $(B=0)$, the time component of the Klein-Gordon equation or the spin diagonal component of the Dirac equation may be written as

$$
\ddot{\varphi}_{\mathbf{k}}(t)+\omega_{\mathbf{k}}^{2}(t) \varphi_{\mathbf{k}}(t)=0
$$

where

$$
\omega_{\mathbf{k}}^{2}(t)=\left(k_{z}-q E_{0} \tau\left(1+\tanh \left(\frac{t}{\tau}\right)\right)\right)^{2}+\mathbf{k}_{\perp}^{2}+m^{2}+2 i \sigma q E(t)
$$

where $\sigma= \pm 1 / 2$ denotes the spin state. The mode equation (53) has the asymptotic frequency at $t=-\infty$ and $+\infty$,

$$
\omega_{\mathbf{k}, \text { in }}=\sqrt{\mathbf{k}^{2}+m^{2}}, \quad \omega_{\mathbf{k}, \text { out }}=\sqrt{\left(k_{z}-2 q E_{0} \tau\right)^{2}+\mathbf{k}_{\perp}^{2}+m^{2}} .
$$

\section{A. Scalar QED}

The Bogoliubov coefficients between the ingoing and the outgoing vacua are found from the solution to Eq. (53) with $\sigma=0$, which has the asymptotic ingoing solution at $t=-\infty$,

$$
\varphi_{\mathbf{k}, \text { in }}(t)=\frac{e^{-i \omega_{\mathbf{k}, \text { in }} t}}{\sqrt{2 \omega_{\mathbf{k}, \text { in }}}} .
$$

The solution is given by [5, 20, 24, 31]

$$
\varphi_{\mathbf{k}}(t)=\frac{1}{\sqrt{2 \omega_{\mathbf{k}, \text { in }} e^{\pi \tau \omega_{\mathbf{k}, \text { in }}}}}(1-z)^{1 / 2+i \lambda^{\mathrm{sc}}} z^{-i \tau \omega_{\mathbf{k}, \text { in }} / 2} F\left(\alpha_{\mathbf{k}}, \beta_{\mathbf{k}} ; \gamma_{\mathbf{k}} ; z\right),
$$

where $F\left(\alpha_{\mathbf{k}}, \beta_{\mathbf{k}} ; \gamma_{\mathbf{k}} ; z\right)$ is the hypergeometric function, and

$$
z=-e^{2 t / \tau}, \quad \lambda^{\mathrm{sc}}=\sqrt{\left(q E_{0} \tau^{2}\right)^{2}-\frac{1}{4}}
$$

and

$$
\begin{aligned}
\alpha_{\mathbf{k}} & =\frac{1}{2}-\frac{i}{2}\left(\tau \omega_{\mathbf{k}, \text { in }}-\tau \omega_{\mathbf{k}, \text { out }}-2 \lambda^{\mathrm{sc}}\right) \\
\beta_{\mathbf{k}} & =\frac{1}{2}-\frac{i}{2}\left(\tau \omega_{\mathbf{k}, \text { in }}+\tau \omega_{\mathbf{k}, \text { out }}-2 \lambda^{\mathrm{sc}}\right) \\
\gamma_{\mathbf{k}} & =1-i \tau \omega_{\mathbf{k}, \text { in }} .
\end{aligned}
$$


The solution evolves to the form at $t=\infty$

$$
\varphi_{\mathbf{k}}(t)=\mu_{\mathbf{k}} \varphi_{\mathbf{k}, \text { out }}(t)+\nu_{\mathbf{k}} \varphi_{\mathbf{k}, \text { out }}^{*}(t),
$$

where

$$
\varphi_{\mathbf{k}, \text { out }}(t)=\frac{e^{-i \omega_{\mathbf{k}, \text { out }} t}}{\sqrt{2 \omega_{\mathbf{k}, \text { out }}}}
$$

is the outgoing solution, and

$$
\begin{aligned}
& \mu_{\mathbf{k}}=2^{-i \tau \omega_{\mathbf{k}, \text { out }}} \sqrt{\frac{\omega_{\mathbf{k}, \text { out }}}{\omega_{\mathbf{k}, \text { in }}}}\left(\frac{\Gamma\left(\gamma_{\mathbf{k}}\right) \Gamma\left(\beta_{\mathbf{k}}-\alpha_{\mathbf{k}}\right)}{\Gamma\left(\beta_{\mathbf{k}}\right) \Gamma\left(\gamma_{\mathbf{k}}-\alpha_{\mathbf{k}}\right)}\right), \\
& \nu_{\mathbf{k}}=2^{i \tau \omega_{\mathbf{k}}^{\text {out }}} \sqrt{\frac{\omega_{\mathbf{k}, \text { out }}}{\omega_{\mathbf{k}, \text { in }}}}\left(\frac{\Gamma\left(\gamma_{\mathbf{k}}\right) \Gamma\left(\alpha_{\mathbf{k}}-\beta_{\mathbf{k}}\right)}{\Gamma\left(\alpha_{\mathbf{k}}\right) \Gamma\left(\gamma_{\mathbf{k}}-\beta_{\mathbf{k}}\right)}\right) .
\end{aligned}
$$

The terms in front of the brackets of the Bogoliubov coefficient (62) are removed in a renormalization procedure; thus, according to Eq. (23), the effective action per unit volume is given by

$$
\mathcal{L}_{\mathrm{eff}}^{\mathrm{sc}}=i \int \frac{d^{3} \mathbf{k}}{(2 \pi)^{3}}\left[\ln \Gamma\left(\gamma_{\mathbf{k}}^{*}\right)+\ln \Gamma\left(\beta_{\mathbf{k}}^{*}-\alpha_{\mathbf{k}}^{*}\right)-\ln \Gamma\left(\beta_{\mathbf{k}}^{*}\right)-\ln \Gamma\left(\gamma_{\mathbf{k}}^{*}-\alpha_{\mathbf{k}}^{*}\right)\right] .
$$

By using the gamma function (42), we obtain the effective action in an intermediate form

$$
\begin{aligned}
\mathcal{L}_{\mathrm{eff}}^{\mathrm{sc}}= & -\frac{i}{2} \int \frac{d^{3} \mathbf{k}}{(2 \pi)^{3}} \int_{0}^{\infty} \frac{d s}{s}\left(e^{-\frac{i}{2} \Omega_{\mathbf{k}}^{(+)} s}+e^{-\frac{i}{2} \Omega_{\mathbf{k}}^{(-)} s}\right)\left[\frac{1}{\sinh (s / 2)}-\frac{2}{s}+\frac{s}{12}\right] \\
& +\frac{i}{2} \int \frac{d^{3} \mathbf{k}}{(2 \pi)^{3}} \int_{0}^{\infty} \frac{d s}{s} e^{-i \tau \omega_{\mathbf{k}, \text { in } s}}\left[\frac{e^{-s / 2}}{\sinh (s / 2)}-\frac{2}{s}+1-\frac{s}{6}\right] \\
& +\frac{i}{2} \int \frac{d^{3} \mathbf{k}}{(2 \pi)^{3}} \int_{0}^{\infty} \frac{d s}{s} e^{-i \tau \omega_{\mathbf{k}, \text { out }} s}\left[\frac{e^{s / 2}}{\sinh (s / 2)}-\frac{2}{s}-1-\frac{s}{6}\right],
\end{aligned}
$$

where

$$
\Omega_{\mathbf{k}}^{( \pm)}=\tau \omega_{\mathbf{k}, \text { in }}+\tau \omega_{\mathbf{k}, \text { out }} \pm 2 \lambda^{\mathrm{sc}} .
$$

Finally, by doing the contour integral over a quarter circle in the fourth quadrant, we obtain the exact one-loop effective action per unit volume

$$
\begin{aligned}
\mathcal{L}_{\mathrm{eff}}^{\mathrm{sc}}= & \frac{1}{2} \int \frac{d^{3} \mathbf{k}}{(2 \pi)^{3}} \mathcal{P} \int_{0}^{\infty} \frac{d s}{s}\left(e^{-\Omega_{\mathbf{k}}^{(+)} s}+e^{-\Omega_{\mathbf{k}}^{(-)} s}\right)\left[\frac{1}{\sin (s)}-\frac{1}{s}-\frac{s}{6}\right] \\
& -\frac{1}{2} \int \frac{d^{3} \mathbf{k}}{(2 \pi)^{3}} \mathcal{P} \int_{0}^{\infty} \frac{d s}{s}\left(e^{-2 \tau \omega_{\mathbf{k}, \text { in }} s}+e^{-2 \tau \omega_{\mathbf{k}, \text { out }} s}\right)\left[\cot (s)-\frac{1}{s}+\frac{s}{3}\right] \\
& +\frac{i}{2} \int \frac{d^{3} \mathbf{k}}{(2 \pi)^{3}} \ln \left[\frac{\left(1+e^{-\pi \Omega_{\mathbf{k}}^{(+)}}\right)\left(1+e^{-\pi \Omega_{\mathbf{k}}^{(-)}}\right)}{\left(1-e^{-2 \pi \tau \omega_{\mathbf{k}, \text { in }}}\right)\left(1-e^{-2 \pi \tau \omega_{\mathbf{k}, \text { out }}}\right)}\right] .
\end{aligned}
$$

It can be shown by a direct calculation that

$$
2 \operatorname{Im}\left(\mathcal{L}_{\text {eff }}^{\mathrm{sc}}\right)=\int \frac{d^{3} \mathbf{k}}{(2 \pi)^{3}} \ln \left(1+\mathcal{N}_{\mathbf{k}}^{\mathrm{sc}}\right),
$$

where

$$
\mathcal{N}_{\mathbf{k}}^{\mathrm{sc}}=\frac{\cosh \left(2 \pi \lambda^{\mathrm{sc}}\right)+\cosh \left(\pi \tau \omega_{\mathbf{k}, \text { out }}-\pi \tau \omega_{\mathbf{k}, \text { in }}\right)}{2 \sinh \left(\pi \tau \omega_{\mathbf{k}, \text { in }}\right) \sinh \left(\pi \tau \omega_{\mathbf{k}, \text { out }}\right)} .
$$

The mean number of created pairs, Eq. (68), agrees with the exact result, Eq. (43), of Ref. [32]. 
In the second case of the electric field together with a constant magnetic field, the mode equation takes the form

$$
\ddot{\varphi}_{k}(t, x, y)+\left[-\left(\partial_{x}^{2}+\partial_{y}^{2}\right)+\left(\frac{q B}{2}\right)^{2}\left(x^{2}+y^{2}\right)+q B L_{z}+\left(k_{z}-q E_{0} \tau\left(1+\tanh \left(\frac{t}{\tau}\right)\right)\right)^{2}+m^{2}\right] \varphi_{k}(t, x, y)=0
$$

Now, the mode equation (69) has the asymptotic frequency,

$$
\omega_{n k, \text { in }}=\sqrt{k_{z}^{2}+q B(2 n+1)+m^{2}}, \quad \omega_{n k, \text { out }}=\sqrt{\left(k_{z}-2 q E_{0} \tau\right)^{2}+q B(2 n+1)+m^{2}} .
$$

In Ref. [24], the solution is found to be $\varphi_{k}(t, x, y)=\varphi_{n k}(t) \varphi_{n}(x, y)$, where $\varphi_{n}(x, y)$ is the two-dimensional harmonic oscillator function with the Landau level, $q B(2 n+1)$, and

$$
\varphi_{n k}(t)=\frac{1}{\sqrt{2 \omega_{n k, \mathrm{in}} e^{\pi \tau \omega_{n k, \mathrm{in}}}}}(1-z)^{1 / 2+i \lambda^{\mathrm{sc}}} z^{-i \tau \omega_{n k, \mathrm{in}} / 2} F\left(\alpha_{n k}, \beta_{n k} ; \gamma_{n k} ; z\right)
$$

with parameters replaced by

$$
\begin{aligned}
\alpha_{n k} & =\frac{1}{2}-\frac{i}{2}\left(\tau \omega_{n k, \text { in }}-\tau \omega_{n k, \text { out }}-2 \lambda^{\mathrm{sc}}\right), \\
\beta_{n k} & =\frac{1}{2}-\frac{i}{2}\left(\tau \omega_{n k, \text { in }}+\tau \omega_{n k, \text { out }}-2 \lambda^{\mathrm{sc}}\right), \\
\gamma_{n k} & =1-i \tau \omega_{n k, \text { in }} .
\end{aligned}
$$

By repeating the same procedure for the pure electric field, we obtain the exact one-loop effective action per unit volume

$$
\begin{aligned}
\mathcal{L}_{\mathrm{eff}}^{\mathrm{sc}}= & \frac{1}{2} \int \frac{d k_{z}}{2 \pi} \mathcal{P} \int_{0}^{\infty} \frac{d s}{s} \sum_{n=0}^{\infty}\left(e^{-\Omega_{n k}^{(+)} s}+e^{-\Omega_{n k}^{(-)} s}\right)\left[\frac{1}{\sin (s)}-\frac{1}{s}+\frac{s}{6}\right] \\
& -\frac{1}{2} \int \frac{d k_{z}}{2 \pi} \mathcal{P} \int_{0}^{\infty} \frac{d s}{s} \sum_{n=0}^{\infty}\left(e^{-2 \tau \omega_{n k, \text { in }} s}+e^{-2 \tau \omega_{n k, \text { out }} s}\right)\left[\cot (s)-\frac{1}{s}+\frac{s}{3}\right] \\
& +\frac{i}{2} \int \frac{d k_{z}}{2 \pi} \sum_{n=0}^{\infty} \ln \left[\frac{\left(1+e^{-\pi \Omega_{n k}^{(+)}}\right)\left(1+e^{-\pi \Omega_{n k}^{(-)}}\right)}{\left(1-e^{-2 \pi \tau \omega_{n k, \text { out }}}\right)\left(1-e^{\left.-2 \pi \tau \omega_{n k, \text { in }}\right)}\right.}\right]
\end{aligned}
$$

where

$$
\Omega_{n k}^{( \pm)}=\tau \omega_{n k, \text { in }}+\tau \omega_{n k, \text { out }} \pm 2 \lambda^{\mathrm{sc}} .
$$

It can be shown that $2 \operatorname{Im}\left(\mathcal{L}_{\text {eff }}^{\mathrm{sc}}\right)=\sum_{\mathbf{k}} \ln \left(1+\mathcal{N}_{\mathbf{k}}^{\mathrm{sc}}\right)$, thus confirming the renormalized relation of Eq. (25).

\section{B. Spinor QED}

The Sauter-type electric field in spinor QED does not change the asymptotic solutions $\varphi_{\mathbf{k} \text {,in }}$ and $\varphi_{\mathbf{k}, \text { out }}$ for each spin diagonal component, which define the ingoing and the outgoing vacua. The spin diagonal component equation (53) has the solution [20, 31]

$$
\varphi_{\mathbf{k}}(t)=\frac{1}{\sqrt{2 \omega_{\mathbf{k}, \text { in }} e^{\pi \tau \omega_{\mathbf{k}, \text { in }}}}}(1-z)^{(1-2 \sigma) / 2+i \lambda^{\mathrm{sp}}} z^{-i \tau \omega_{\mathbf{k}, \text { in }} / 2} F\left(\alpha_{\mathbf{k}}^{\mathrm{sp}}, \beta_{\mathbf{k}}^{\mathrm{sp}} ; \gamma_{\mathbf{k}} ; z\right)
$$

where

$$
z=-e^{2 t / \tau}, \quad \lambda^{\mathrm{sp}}=q E_{0} \tau^{2}
$$

and

$$
\begin{aligned}
\alpha_{\mathbf{k}}^{\mathrm{sp}} & =\frac{1-2 \sigma}{2}-\frac{i}{2}\left(\tau \omega_{\mathbf{k}, \text { in }}-\tau \omega_{\mathbf{k}, \text { out }}-2 \lambda^{\mathrm{sp}}\right) \\
\beta_{\mathbf{k}}^{\mathrm{sp}} & =\frac{1-2 \sigma}{2}-\frac{i}{2}\left(\tau \omega_{\mathbf{k}, \text { in }}+\tau \omega_{\mathbf{k}, \text { out }}-2 \lambda^{\mathrm{sp}}\right) \\
\gamma_{\mathbf{k}} & =1-i \tau \omega_{\mathbf{k}, \text { in }} .
\end{aligned}
$$


Then the Bogoliubov coefficients are

$$
\begin{aligned}
& \mu_{\mathbf{k}}^{\mathrm{sp}}=2^{-i \tau \omega_{\mathbf{k}, \text { out }}} \sqrt{\frac{\omega_{\mathbf{k}, \text { out }}}{\omega_{\mathbf{k}, \text { in }}}}\left(\frac{\Gamma\left(\gamma_{\mathbf{k}}\right) \Gamma\left(\beta_{\mathbf{k}}^{\mathrm{sp}}-\alpha_{\mathbf{k}}^{\mathrm{sp}}\right)}{\Gamma\left(\beta_{\mathbf{k}}^{\mathrm{sp}}\right) \Gamma\left(\gamma_{\mathbf{k}}-\alpha_{\mathbf{k}}^{\mathrm{sp}}\right)}\right), \\
& \nu_{\mathbf{k}}^{\mathrm{sp}}=2^{i \tau \omega_{\mathbf{k}}^{\text {out }}} \sqrt{\frac{\omega_{\mathbf{k}, \text { out }}}{\omega_{\mathbf{k}, \text { in }}}}\left(\frac{\Gamma\left(\gamma_{\mathbf{k}}\right) \Gamma\left(\alpha_{\mathbf{k}}^{\mathrm{sp}}-\beta_{\mathbf{k}}^{\mathrm{sp}}\right)}{\Gamma\left(\alpha_{\mathbf{k}}^{\mathrm{sp}}\right) \Gamma\left(\gamma_{\mathbf{k}}-\beta_{\mathbf{k}}^{\mathrm{sp}}\right)}\right)
\end{aligned}
$$

and the effective action per unit volume is given by

$$
\mathcal{L}_{\mathrm{eff}}^{\mathrm{sp}}=i \int \frac{d^{3} \mathbf{k}}{(2 \pi)^{3}}\left[\ln \Gamma\left(\gamma_{\mathbf{k}}^{*}\right)+\ln \Gamma\left(\beta_{\mathbf{k}}^{\mathrm{sp} *}-\alpha_{\mathbf{k}}^{\mathrm{sp} *}\right)-\ln \Gamma\left(\beta_{\mathbf{k}}^{\mathrm{sp} *}\right)-\ln \Gamma\left(\gamma_{\mathbf{k}}-\alpha_{\mathbf{k}}^{\mathrm{sp} *}\right)\right] .
$$

Finally, using the gamma function (42) and summing over the spin states, we obtain the exact one-loop effective action in spinor QED

$$
\begin{aligned}
\mathcal{L}_{\mathrm{eff}}^{\mathrm{sp}}= & -\int \frac{d^{3} \mathbf{k}}{(2 \pi)^{3}} \mathcal{P} \int_{0}^{\infty} \frac{d s}{s}\left[\left(e^{-\Omega_{\mathbf{k}}^{\mathrm{sp}(+)} s}+e^{-\Omega_{\mathbf{k}}^{\mathrm{sp}(-)} s}\right)-\left(e^{-2 \tau \omega_{\mathbf{k}, \text { in }} s}+e^{-2 \tau \omega_{\mathbf{k}, \text { out }} s}\right)\right]\left(\cot (s)-\frac{1}{s}+\frac{s}{3}\right) \\
& -i \int \frac{d^{3} \mathbf{k}}{(2 \pi)^{3}} \ln \left[\frac{\left(1-e^{-\pi \Omega_{\mathbf{k}}^{\mathrm{sp}(+)}}\right)\left(1-e^{-\pi \Omega_{\mathbf{k}}^{\mathrm{sp}(-)}}\right)}{\left(1-e^{-2 \pi \tau \omega_{\mathbf{k}, \text { in }}}\right)\left(1-e^{\left.-2 \pi \tau \omega_{\mathbf{k}, \text { out }}\right)}\right.}\right],
\end{aligned}
$$

where

$$
\Omega_{\mathbf{k}}^{\mathrm{sp}( \pm)}=\tau \omega_{\mathbf{k}, \text { in }}+\tau \omega_{\mathbf{k}, \text { out }} \pm 2 \lambda^{\mathrm{sp}} .
$$

Note that the effective action (80) is finite due to the renormalization of vacuum-energy and charge. The difference of the factor of two from Eq. (66) in scalar QED is the spin multiplicity of spin-1/2 fermions. A direct calculation leads to the general relation, which is renormalized,

$$
\operatorname{Im}\left(\mathcal{L}_{\mathrm{eff}}^{\mathrm{sp}}\right)=-\int \frac{d^{3} \mathbf{k}}{(2 \pi)^{3}} \ln \left(1-\mathcal{N}_{\mathbf{k}}^{\mathrm{sp}}\right)
$$

where

$$
\mathcal{N}_{\mathbf{k}}^{\mathrm{sp}}=\frac{\cosh \left(2 \pi \lambda^{\mathrm{sp}}\right)-\cosh \left(\pi \tau \omega_{\mathbf{k}, \text { out }}-\pi \tau \omega_{\mathbf{k}, \text { in }}\right)}{2 \sinh \left(\pi \tau \omega_{\mathbf{k}, \text { in }}\right) \sinh \left(\pi \tau \omega_{\mathbf{k}, \text { out }}\right)} .
$$

A few comments are in order. First, the mean number (83) from the imaginary part agrees with the exact result (42) for spin-1/2 fermions of Ref. [32] and also with Ref. [31]. Second, we may compare the imaginary part with Eq. (30) of Ref. [14],

$$
\operatorname{Im}\left(\mathcal{L}_{\mathrm{eff}}^{\mathrm{sp}}\right)=\int \frac{d^{3} \mathbf{k}}{(2 \pi)^{3}} \ln \left[\left(1-e^{-\pi \Omega_{\mathbf{k}}^{(+)}}\right)\left(1-e^{-\pi \Omega_{\mathbf{k}}^{(-)}}\right)\right] .
$$

However, the denominator of the imaginary part in Eq. (80) is necessary to explain no pair production in the limit of either $E_{0}=0$ for a finite $\tau$ or $\tau=0$ for a finite $E_{0}$. Note that in the limit of $\tau \omega_{\mathbf{k} \text {,in }} \gg 1$ the denominator approaches to identity and the imaginary part of Eq. (80) reduces to Eq. (84).

In the presence of a constant magnetic field parallel to the Sauter-type electric field, the exact one-loop effective action is obtained by summing over Landau levels,

$$
\begin{aligned}
\mathcal{L}_{\mathrm{eff}}^{\mathrm{sp}}= & -\int \frac{d k_{z}}{2 \pi} \mathcal{P} \int_{0}^{\infty} \frac{d s}{s} \sum_{n=0}^{\infty}\left[\left(e^{-\Omega_{n k}^{\mathrm{sp}(+)} s}+e^{-\Omega_{n k}^{\mathrm{sp}(-)} s}\right)-\left(e^{-2 \tau \omega_{n k, \text { in }} s}+e^{-2 \tau \omega_{n k, \text { out }} s}\right)\right]\left(\cot (s)-\frac{1}{s}+\frac{s}{3}\right) \\
& -i \int \frac{d k_{z}}{2 \pi} \mathcal{P} \int_{0}^{\infty} \frac{d s}{s} \sum_{n=0}^{\infty} \ln \left[\frac{\left(1-e^{-\pi \Omega_{n k}^{\mathrm{sp}(+)}}\right)\left(1-e^{-\pi \Omega_{n k}^{\mathrm{sp}(-)}}\right)}{\left(1-e^{-2 \pi \tau \omega_{n k, \text { in }}}\right)\left(1-e^{\left.-2 \pi \tau \omega_{n k, \text { out }}\right)}\right]}\right.
\end{aligned}
$$

where

$$
\omega_{n k, \text { in }}=\sqrt{k_{z}^{2}+q B(2 n+1)+m^{2}}, \quad \omega_{n k, \text { out }}=\sqrt{\left(k_{z}-2 q E_{0} \tau\right)^{2}+q B(2 n+1)+m^{2}} .
$$

and

$$
\Omega_{n k}^{\mathrm{sp}( \pm)}=\tau \omega_{n k, \text { in }}+\tau \omega_{n k, \text { out }} \pm 2 \lambda^{\mathrm{sp}}
$$




\section{CONCLUSION}

In this paper we further developed the evolution operator method to calculate the effective action of scalar and spinor QED in electric field backgrounds, which was expressed in terms of the Bogoliubov coefficient. In fact, the Bogoliubov transformation between the ingoing and the outgoing Fock space enables one to explicitly calculate the scattering amplitude between the ingoing and the outgoing vacua, whose complex phase is the effective action. For that purpose, we first expressed the evolution operator in terms of a two-mode squeeze operator and an overall phase part. As the two-mode squeeze operator carries the information about the multi-pair production, this approach correctly yields not only the mean number of created pairs but also the vacuum polarization.

We first applied the method to a constant electric field to clarify the renormalization procedure. The exact one-loop effective action and its imaginary part thus obtained agreed with the standard scalar and spinor QED result from other methods. According to the electric-magnetic duality, the Landau levels of a charged scalar particle or spin- $1 / 2$ fermion in a magnetic field correspond to a discrete spectrum of complex frequencies in the electric field. However, the actual motion in an electric field is an acceleration. Instead, the evolution operator method makes use of the Bogoliubov transformation between the ingoing and the outgoing Fock space. The Bogoliubov coefficient leads to the exact one-loop effective action simultaneously with the correct imaginary part as the sum of residues of a proper integral.

As the next application, we applied the method to a Sauter-type electric field, effectively acting for a finite period of time. Using the Bogoliubov coefficient, we obtained the exact one-loop effective action at zero temperature, which is the main result of this paper. The real part of the effective action of scalar and spinor QED takes the form

$$
\operatorname{Re}\left(\mathcal{L}_{\mathrm{eff}}\right)= \pm \frac{2 S+1}{2} \int \frac{d^{3} \mathbf{k}}{(2 \pi)^{3}} \mathcal{P} \int_{0}^{\infty} \frac{d s}{s}\left[\left(e^{-\Omega_{\mathbf{k}}^{(+)} s}+e^{-\Omega_{\mathbf{k}}^{(-)} s}\right) f(s)-\left(e^{-2 \tau \omega_{\mathbf{k}, \text { in }} s}+e^{-2 \tau \omega_{\mathbf{k}, \text { out }} s}\right) g(s)\right]
$$

where the upper (lower) sign and $S=0(1 / 2)$ are for scalar particles (spin- $1 / 2$ fermions), and $g(s)=\cot (s)-1 / s+s / 3$, and $f(s)=1 / \sin (s)-1 / s+s / 6$ for scalar particles and $f(s)=g(s)$ for spin- $1 / 2$ fermions. The imaginary part is given by

$$
\operatorname{Im}\left(\mathcal{L}_{\text {eff }}\right)= \pm \frac{2 S+1}{2} \int \frac{d^{3} \mathbf{k}}{(2 \pi)^{3}} \ln \left[\frac{\left(1 \pm e^{-\pi \Omega_{\mathbf{k}}^{(+)}}\right)\left(1 \pm e^{-\pi \Omega_{\mathbf{k}}^{(-)}}\right)}{\left(1-e^{-2 \pi \tau \omega_{\mathbf{k}, \text { in }}}\right)\left(1-e^{-2 \pi \tau \omega_{\mathbf{k}, \text { out }}}\right)}\right]
$$

where the upper positive signs are for scalar particles and the lower negative signs for spin- $1 / 2$ fermions. It is further shown that the imaginary part of the renormalized effective action indeed satisfies the general relation, as expected,

$$
2\left(\operatorname{Im} \mathcal{L}_{\text {eff }}\right)= \pm(2 S+1) \int \frac{d^{3} \mathbf{k}}{(2 \pi)^{3}} \ln \left(1 \pm \mathcal{N}_{\mathbf{k}}\right)
$$

where

$$
\mathcal{N}_{\mathbf{k}}=\frac{\cosh (2 \pi \lambda) \pm \cosh \left(\pi \tau \omega_{\mathbf{k}, \text { out }}-\pi \tau \omega_{\mathbf{k}, \text { in }}\right)}{2 \sinh \left(\pi \tau \omega_{\mathbf{k}, \text { in }}\right) \sinh \left(\pi \tau \omega_{\mathbf{k}, \text { out }}\right)}
$$

The mean number of created pairs, Eq. (91), agrees with the exact results, Eq. (43) for scalar particles and Eq. (42) for spin-1/2 fermions of Ref. [32]. The denominator in Eq. (89), which is missing in Ref. [14], is necessary to explain no pair production in the limit of either $E_{0}=0$ for a finite $\tau$ or $\tau=0$ for a finite $E_{0}$. However, in the other limit of $\tau \rightarrow 0$ for a finite $E_{0} \tau$, the mean number approaches the limiting value $\mathcal{N}_{\mathbf{k}}^{\mathrm{sc}}=\left(\omega_{\mathbf{k} \text {,out }}-\omega_{\mathbf{k}, \text { in }}\right)^{2} /\left(4 \omega_{\mathbf{k} \text {,in }} \omega_{\mathbf{k} \text {,out }}\right)$ for scalar particles and $\mathcal{N}_{\mathbf{k}}^{\mathrm{sp}}=\left[4\left(q E_{0} \tau\right)^{2}-\left(\omega_{\mathbf{k}, \text { out }}-\omega_{\mathbf{k}, \text { in }}\right)^{2}\right] /\left(4 \omega_{\mathbf{k}, \text { in }} \omega_{\mathbf{k} \text {,out }}\right)$ for spin- $1 / 2$ fermions. Finally, we found the exact one-loop effective action both in the Sauter-type electric field and in a constant magnetic field.

It should be pointed out that the method can be applied to the finite-temperature effective action in a timedependent electric field, which describes a nonequilibrium quantum field [33].

\section{Acknowledgments}

We would like to thank Prof. S. P. Gavrilov for useful comments. S. P. K. appreciates the hospitality of Hanyang University and Center for Quantum Spacetime (CQUeST) of Sogang University, and H. K. L. and Y. Y. appreciate the hospitality of Kunsan National University. The work of S. P. K. was supported by the Korea Research Foundation Grant funded by the Korean Government (MOEHRD) (KRF-2007-C00167) and the work of H. K. L. was supported 
by the Korea Science and Engineering Foundation (KOSEF) grant funded by the Korea government (MOST) (No. R01-2006-000-10651-0).

[1] F. Sauter, Z. Phys. 69, 742 (1931); W. Heisenberg and H. Euler, Z. Physik 98, 714 (1936); V. Weisskopf, K. Dan. Vidensk. Selsk. Mat. Fys. Medd. XIV, No. 6 (1936).

[2] J. Schwinger, Phys. Rev. 82, 664 (1951).

[3] G. V. Dunne, "Heisenberg-Euler Effective Lagrangians: Basics and Extensions," From Fields to Strings: Circumnavigating Theoretical Physics, edited by M. Shifman, A. Vainshtein, and J. Wheater, (World Scientific, Singapore, 2005), Vol. I, pp. 445-522, hep-th/0406216.

[4] A. Ringwald, Phys. Lett. B 510, 107 (2001); "Fundamental Physics at an X-Ray Free Electron Laser," Electromagnetic Probes of Fundamental Physics, edited by W. Marciano and S. White (World Scientific, Singapore, 2003), pp. 63-74, hep-ph/0112254 "Boiling the Vacuum with an X-Ray Free Electron Laser," Quantum Aspects of Beam Physics 2003, edited by P. Chen and K. Reil (World Scientific, Singapore, 2004), pp. 149-163, hep-ph/0304139

[5] S. P. Kim and D. N. Page, Phys. Rev. D 65, 105002 (2002).

[6] S. P. Kim and D. N. Page, Phys. Rev. D 73, 065020 (2006).

[7] S. P. Kim and D. N. Page, Phys. Rev. D 75, 045013 (2007).

[8] H. Gies and K. Klingmüller, Phys. Rev. D 72, 065001 (2005).

[9] G. V. Dunne and C. Schubert, Phys. Rev. D 72, 105004 (2005).

[10] G. V. Dunne, Q.-H. Wang, H. Gies, and C. Schubert, Phys. Rev. D 73, 065028 (2006).

[11] H. Kleinert, R. Ruffini, and S.-S. Xue, Phys. Rev. D 78, 025011 (2008).

[12] Y. M. Cho, and D. G. Pak, Phys. Rev. Lett. 86, 1947 (2001); W. S. Bae, Y. M. Cho, and D. G. Pak, Phys. Rev. D 64, 017303 (2001).

[13] R. Brout, R. Parentani, and Ph. Spindel, Nucl. Phys. B 353, 209 (1991); R. Parentani and R. Brout, Nucl. Phys. B 388, 474 (1992); R. Parentani and S. Massar, Phys. Rev. D 55, 3603 (1997).

[14] G. Dunne and T. Hall, Phys. Rev. D 58, 105022 (1998).

[15] H. M. Fried and R. P. Woodard, Phys. Lett. B 524, 233 (2002).

[16] S. P. Kim and H. K. Lee, "Effective Action and Schwinger Pair Production in Scalar QED," arXiv: 0806.2496 (2008).

[17] J. Schwinger, Proc. Nat. Acad. Sci. 37, 452 (1951).

[18] A. I. Nikishov, Zh. Eksp. Teor. Fiz 57, 1210 (1970) [Sov. Phys. JETP 30, 660 (1970)].

[19] B. S. DeWitt, Phys. Rep. 19, 295 (1975).

[20] J. Ambjorn, R. J. Hughes, and N. K. Nielsen, Ann. Phys. 150, 92 (1983).

[21] A. I. Nikishov, JETP 96, 180 (2003).

[22] E. S. Fradkin, D. M. Gitman, and S. M. Shvartsman, Quantum Electrodynamics with Unstable Vacuum (Springer-Verlag, Berlin, 1991).

[23] S. P. Gavrilov, D. M. Gitman, and J. L. Tomazelli, Nucl. Phys. B 795, 645 (2008).

[24] S. P. Kim and H. K. Lee, Phys. Rev D 76, 125002 (2007).

[25] C. M. Caves and B. L. Schumaker, Phys. Rev. A 31, 3068 (1985).

[26] B. L. Schumaker and C. M. Caves, Phys. Rev. A 31, 3093 (1985).

[27] H.-Y. Fan and C. Li, J. Opt. B: Quantum Semiclass. Opt. 6, S502 (2004).

[28] I. S. Gradshteyn and I. M. Ryzhik, Table of Integrals, Series, and Products (Academic Press, San Diego, 1994), 9.248-2.

[29] I. S. Gradshteyn and I. M. Ryzhik, Table of Integrals, Series, and Products (Academic Press, San Diego, 1994), 8.341-3.

[30] F. Sauter, Z. Phys. 73, 547 (1932).

[31] N. B. Narozhnyi and A. I. Nikishov, Yad. Fiz. 11, 1072 (1970) [Sov. J. Nucl. Phys. 11, 596 (1970)].

[32] S. P. Gavrilov and D. M. Gitman, Phys. Rev. D 53, 7162 (1996).

[33] S. P. Kim, H. K. Lee, and Y. Yoon, in preparation. 